\title{
The Role of MMP-9 and VEGF in the Invasion State of Bladder Urothelial Carcinoma
}

\author{
Novalia Chumaladewi Guntarno ${ }^{1,2,3}$, Anny Setijo Rahaju ${ }^{1,2,3 *}$, Nila Kurniasari ${ }^{1,2,3}$ \\ ${ }^{1}$ Department of Anatomical Pathology, Faculty of Medicine, Universitas Airlangga, Jl. Mayjen Prof. Dr. Moestopo No.47, Surabaya, Indonesia \\ ${ }^{2}$ Universitas Airlangga Hospital, Jl. Dharmahusada Permai No.116, Surabaya, Indonesia \\ ${ }^{3}$ Dr. Soetomo General Academic Hospital, Jl. Mayjen Prof. Dr. Moestopo No.6-8, Surabaya, Indonesia \\ *Corresponding author. E-mail: anny_sr@fk.unair.ac.id
}

Received date: Jul 22, 2020; Revised date: Dec 31, 2020; Accepted date: Jan 4, 2021

\section{Abstract}

B ACKGROUND: Bladder cancer is the tenth-most common cancer in worldwide and the majority are urothelial carcinomas. The depth of invasion plays important role in the prognostic and therapeutic factor in urothelial carcinomas. The expression of vascular endothelial growth factor (VEGF) and matrix metalloproteinase-9 (MMP-9) may associated with the progression of bladder carcinoma, such as depth of invasion. In this study, the correlation between these two markers in urothelial bladder carcinoma invasion will be analyzed.

METHODS: An analytical observational research with cross-sectional were conducted on 54 formalin fixed paraffin-embedded tissue from radical cystectomy (RC) which were diagnosed as bladder urothelial carcinoma at the Anatomical Pathology Laboratory of Dr. Soetomo Academic Hospital, Surabaya and divided based on the $\mathrm{T}$ stage were immunostained using VEGF and MMP-9 monoclonal antibodies. The difference of VEGF and MMP-

\section{Introduction}

Bladder carcinoma is the ninth malignancy worldwide and seventh of the most common malignancy in men. Based on Global Cancer Statistic (GLOBOCAN) 2018, bladder carcinoma was the tenth malignancy with 549.000 cases and 200.000 cases of mortality rate.(1)
9 expression in $\mathrm{T}$ stage of bladder urothelial carcinoma were analyzed using Kruskal-Wallis and Anova test, the correlation between VEGF and MMP-9 expression in various $\mathrm{T}$ stage of bladder urothelial carcinoma were analyzed using Spearman test.

RESULTS: This study showed no significant difference of VEGF expression among $\mathrm{T}$ stage of bladder urothelial carcinoma $(p>0.05)$ but there was significant difference of MMP-9 expression in T stage of bladder urothelial carcinoma $(p=0.043)$. There was a correlation between VEGF and MMP-9 in various T stage of bladder urothelial carcinoma $(\mathrm{rs}=0.50, p=0.001)$.

CONCLUSION: The significant correlation of VEGF and MMP-9 expression in bladder urothelial carcinoma may prove the synergistically role of both proteins in tumor invasion by MMP-9 degradation extracellular matrix.

KEYWORDS: urothelial carcinoma, VEGF, MMP-9, T stage

Indones Biomed J. 2021; 13(1): 61-7

Urothelial cell carcinoma is the most common histopathology subtype in bladder carcinoma, accounting for about $90 \%$ of all bladder malignancies.(2) Determination of the degree of invasive tumor in bladder carcinoma is very important both for prognosis and the determination of therapy. T1 stage is stage of bladder carcinoma that has not yet penetrated to the muscular layer has a recurrence rate of 50 to $70 \%$ with good prognosis and the management 
of the treatment is provided by cystoscopy resection and intravesicular therapy radical cystectomy (RC) can be performed in $\mathrm{T} 1$ stage with high risk condition such as tumor size $>3 \mathrm{~cm}$ and multiple tumors. While T2, T3, T4 stage are stage of bladder carcinoma that has penetrated the muscular layer has a worse prognosis with combination therapy between cystectomy and cisplatin-based combination chemotherapy.(3-6) Therefore, it is important to analyze the factors that play a role in bladder carcinoma invasion.

One of the most important factors in tumor growth and invasion is angiogenesis. Rapid tumor growth increases the need for oxygen and nutrients for metabolic processes. Tumor cells require the formation of new blood vessels to deliver oxygen and nutrients to tumor cells that are very proliferative.(7) Vascular endothelial growth factor (VEGF) is one of the angiogenic factor that important to stimulates angiogenesis and tumor growth family of growth factors and the VEGF receptor forms a tumor angiogenesis signal pathway. The significance of the prognosis of VEGF expression has been studied in various types of cancer and several studies state that VEGF has an important role in bladder cancer progression and invasion.(8-10)

The basis of angiogenesis is the migration of endothelial cells into the surrounding loose tissue. Matrix metalloproteinases (MMPs) has complex roles including degradation of extracellular matrix, release of proangiogenic substances such as VEGF play a role in facilitating tumor angiogenesis.(11,12) Matrix metalloproteinase-9 (MMP9) is the type of MMP that is involves in the process of degradation of basement membrane and the formation of new blood vessels are characteristic of the progression in bladder carcinoma.(12) Degradation of basement membrane is the key of tumor invasion which is supported by angiogenesis to support the oxygen and nutrient needs. Therefore, this study was conducted to analyze the role and the relationship between VEGF and MMP-9 in invasion (T stage) bladder urothelial carcinoma.

\section{Methods}

\section{Research Design and Sample}

This study had been approved by the Health Research Ethic Committee of Dr. Soetomo Academic Hospital, Surabaya (Ethical Clearance No. 1851/KEPK/II/2020). An analytical observational research with cross-sectional approach were conducted on 54 formalin fixed paraffin-embedded tissue from $\mathrm{RC}$ which were diagnosed as bladder urothelial carcinoma at the Anatomical Pathology Laboratory of Dr. Soetomo Academic Hospital, Surabaya during January 2010 to August 2019. The samples of bladder urothelial carcinoma were divided based on the $\mathrm{T}$ stage (T1, T2, T3, T4) (Table 1).

\section{Immunohistochemistry Staining}

The paraffin blocks of samples were cut into $4 \mu \mathrm{m}$ sections with Leica microtome into slides, deparaffinized three times with xylol for 5 minutes each, and rehydrated through graded alcohol $(96 \%, 96 \%, 90 \%, 80 \%)$ for 2 minutes each. To reduce nonspecific staining due to peroxidased block, the slides were incubated in hydrogen peroxide for 10-15 minutes. Antigen retrieval was achieved by microwaves treatment in sodium citrate buffer ( $\mathrm{pH}$ 6.0) for 45 minutes at $95^{\circ} \mathrm{C}$, then applied background sniper, Biogear - Excell Block, (GitHub, San Francisco, CA, USA). The slides then were incubated with monoclonal antibodies for VEGF (C-1 - sc 7269 dilution 1:200; Santa Cruz Biotechnology, Dallas, TX, USA) and MMP-9 (7-11C - sc 13520 dilution 1:200; Santa Cruz Biotechnology) overnight, and washed in phosphates buffer saline. Secondary antibody, Biogear Universal HRP Excell Stain System - Biogear, BDK-HES125 (GitHub) then applied for 15-20 minutes at room temperature followed 3,3'-diaminobenzidine (DAB) chromogen for 5-15 minutes. Slides then were counterstained with Meyer's hematoxylin and dehydrated with $95 \%$ alcohol.

\section{Evaluation of Immunohistochemical Expression}

The expression of VEGF and MMP-9 was then evaluated using light microscopes Olympus CX41RF at 400x (Olympus, Tokyo, Japan) magnification and documented using Olympus DP2-BSW (Olympus). The results of VEGF expression were interpreted according to the percentage and intensity of immunoreactive product in the cytoplasm of tumor cells. The percentage of tumor cell positively stained was determine by counting the tumor cell that had the highest immunoreactivity. Less than $10 \%$ was scored 0 , more than $10 \%$ to $25 \%$ indicated score 1 , more than $25 \%$ but less than $50 \%$ was scored 2 and more than $50 \%$ of staining was scored 3 . The staining intensity were scored as 1,2,3 for weak, moderate and strong. Then the extend and intensity score were summed as total score, 0-2, 3-4, 5-6 were considered as negative, positive and strong positive.(13)

The results of MMP-9 expression were interpreted according to the percentage of immunoreactive product in the cytoplasm of tumor cells. When the tumor cells 
Table 1. T Stages in urothelial bladder carcinoma.(3)

\begin{tabular}{ll}
\hline & \multicolumn{1}{c}{ T Stages } \\
\hline T1 & Tumor invades the submucosa or lamina propria \\
T2 & Tumor invades muscle tissue \\
T3 & Tumor extends beyond muscle into the perivesical fat \\
T4 & Tumor invades the prostate, vagina, uterus, or bowel, or is fixed to abdominal wall, pelvic wall, or other organs \\
\hline
\end{tabular}

showed less than 5\% indicated negative, more than 5\% to $25 \%$ indicated low levels, more than $25 \%$ but less than $50 \%$ intermediate and more than 50 high levels of staining indicated high.(14)

\section{Statistical Analysis}

All statistical analyses were calculated using SPSS version 25.0 (IBM Corporation, Armonk, NY, USA). The comparison of VEGF was tested using Mann-Whitney U test and MMP-9 using Anova test. The correlation between VEGF and MMP-9 was analyzed using Spearman test with $p<0.05$.

\section{Results}

The average age of the sample in this study was 60.59 years. The youngest age at diagnosis was 34 years while the oldest was 81 years old. The distribution of samples based on age groups was divided into 5 groups with a span of 10 years. The highest number of samples was found in the age group 41-50 years with 13 samples (32.5\%), followed by the 5160 years' age group with 10 samples $(25 \%)$. The gender distribution was 50 samples $(92.59 \%)$ from male patients and 4 samples from female patients. In this study, it was found that the most cases were stage T4 with 16 cases (29.62\%), followed by stages T2 and T3 with the same number of 14 cases $(25.92 \%)$ and stage T1 as many as 10 cases (18.51\%) (Table 2).

\section{VEGF Expression in Bladder Urothelial Carcinoma}

Positive immunoreaction was observed as a brown color staining localized in the cell cytoplasm. There was no significance difference of VEGF expression was found in various $\mathrm{T}$ stage of bladder urothelial carcinoma $p=0.322$ $(p>0.05)$ (Table 3). This study found that VEGF expression in the T1 stage samples had a final score that was evenly distributed from negative to strong positive expressions. Half of the samples in stages T2 and T3 were expressed with score 3 with VEGF and most of the samples in stages T4 were expressed with score 5-6 (Figure 1).

\section{MMP-9 Expression in Bladder Urothelial Carcinoma}

Positive immunoreaction was observed as a brown color staining localized in the cell cytoplasm. There was a significance difference of MMP-9 expression in various T stage of bladder urothelial carcinoma $p=0.043 \quad(p<0.05)$ (Table 4). MMP-9 expression at T1 stage in this study were expressed score 3 in 50\% cases (Figure 2). The data distribution of the MMP-9 expression group was normal, so it was followed by LSD test to find out which group had the most significant difference. The LSD test showed that the T3 and T4 groups were the groups that had the most significant difference with $p=0.008$ and the T2 and $\mathrm{T} 4$ groups with $p=0.04$. The other groups did not have a significant difference (Table 5).

Table 2. The clinicopathological characteristic of subjects.

\begin{tabular}{cc}
\hline \multicolumn{1}{c}{ Characteristics } & n (\%) \\
\hline Age (years)* & $2(3.7)$ \\
$\leq 40$ & $6(11.1)$ \\
$41-50$ & $14(25.9)$ \\
$51-60$ & $26(48.1)$ \\
$61-70$ & $5(9.3)$ \\
$71-80$ & $1(1.9)$ \\
$\geq 81$ & \\
\hline Gender & $50(92.6)$ \\
Male & $4(7.4)$ \\
Female & \\
\hline Tumor grade & $7(13.0)$ \\
Low & $47(87.0)$ \\
High & \\
\hline T stage & $10(18.5)$ \\
T1 & $14(25.9)$ \\
T2 & $14(25.9)$ \\
T3 & $16(29.6)$ \\
T4 &
\end{tabular}


Table 3. VEGF expression in various $\mathrm{T}$ stage bladder urothelial carcinoma.

\begin{tabular}{|c|c|c|c|c|c|}
\hline T Stage & & T1 & $\mathbf{T} 2$ & T3 & T4 \\
\hline \multirow[t]{4}{*}{ Percentage } & $0-10 \% \quad($ Score 0$)$ & $2(20 \%)$ & $2(14.3 \%)$ & 0 & $1(6.2 \%)$ \\
\hline & $10-25 \%($ Score 1$)$ & $2(20 \%)$ & 0 & $5(35.7 \%)$ & $1(6.2 \%)$ \\
\hline & $25-50 \%($ Score 2$)$ & $2(20 \%)$ & $1(7.2 \%)$ & $4(28.6 \%)$ & $3(18.7 \%)$ \\
\hline & $>50 \%($ Score 3$)$ & $6(60 \%)$ & $11(78.5 \%)$ & $5(35.7 \%)$ & $11(6.9 \%)$ \\
\hline \multirow[t]{3}{*}{ Intensity } & Weak (Score 1) & $5(50 \%)$ & $6(42.8 \%)$ & $6(42.8 \%)$ & $7(43.7 \%)$ \\
\hline & Moderate (Score 2) & $4(40 \%)$ & $5(35.7 \%)$ & $5(35.7 \%)$ & $7(43.7 \%)$ \\
\hline & Strong (Score 3) & $1(10 \%)$ & $3(21.4 \%)$ & $3(21.4 \%)$ & $2(12.4 \%)$ \\
\hline \multirow[t]{3}{*}{ Total Score } & Negative (Score $0-2$ ) & $3(30 \%)$ & $2(14.2 \%)$ & $4(28.5 \%)$ & $2(12.4 \%)$ \\
\hline & Positive (Score 3-4) & $3(30 \%)$ & $5(35.7 \%)$ & $3(21.4 \%)$ & $7(43.7 \%)$ \\
\hline & Strong positive (Score 5-6) & $4(40 \%)$ & $7(50 \%)$ & $7(50 \%)$ & $7(43.7 \%)$ \\
\hline$p$-value* (Total Score) & 0.322 & & & & \\
\hline
\end{tabular}

*Tested with Kruskal-Wallis.

\section{Correlation between VEGF and MMP-9 in Bladder Urothelial Carcinoma}

The correlation between VEGF and MMP-9 expression in bladder urothelial carcinoma was significant positive correlation $\mathrm{rs}=0.50 ; p=0.001(p<0.05)$.

\section{Discussion}

This study found that VEGF expression in the 10 samples of T1 stage had final scores that were distributed from negative to strongly positive expressions. Half of the samples in stage T2 (7 samples) and T3 (7 samples) were strongly positive and most of the samples in stages T4 were strongly positive. Factors that can affect VEGF expression with $T$ stage are VEGF receptors (VEGFR-1 and VEGFR-2), which are correlated with tumor invasion in bladder urothelial carcinoma $(p=0.01) .(8)$ The most important interaction between VEGFR-2 and VEGF is the process of mitogenic, chemotactic, angiogenic and the increased permeability effects of VEGF. VEGF and VEGFR-2 bonds can trigger different signaling pathways, for example activation of the
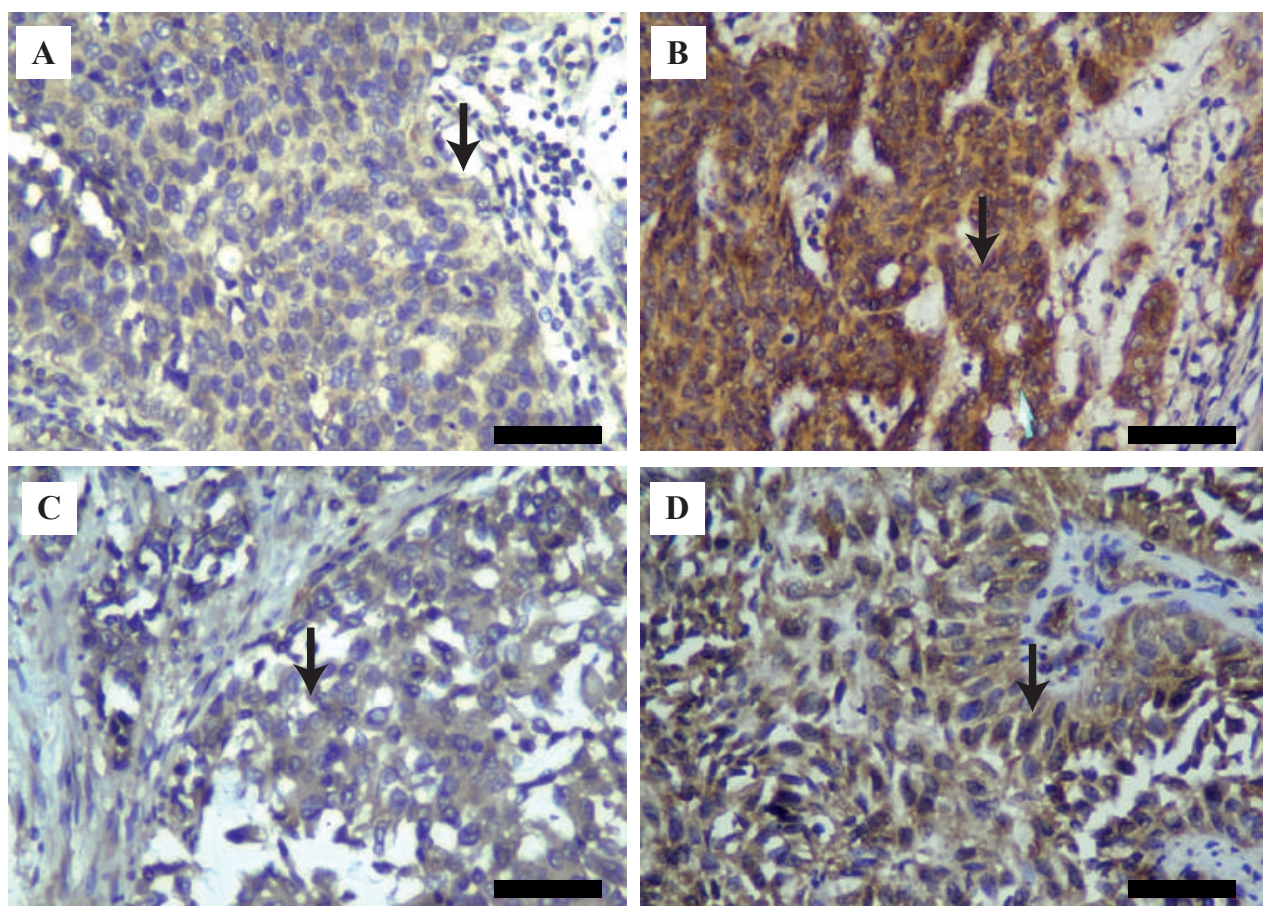

Figure 1. Immunohistochemical expression of VEGFin various T stage of bladder urothelial carcinoma. A: T1 stage, $90 \%$ tumor cells were stained with weak intensity; B: T2 Stage, 65\% tumor cell were stained with strong intensity; C: T3 stage, 70\% tumor cells were stained with weak intensity; D: T4 stage, $95 \%$ tumor cells were stained with moderate intensity. Black bar: $5 \mu \mathrm{m}$. 
Table 4. MMP-9 expression in various T stage bladder urothelial carcinoma.

\begin{tabular}{llcccc}
\hline T Stage & T1 & T2 & T3 & T4 \\
\hline Percentage & $0-5 \%$ (Score 0) & $1(10 \%)$ & 0 & 0 & 0 \\
& $5-25 \%($ Score 1) & $1(10 \%)$ & $5(35.7 \%)$ & $1(7.2 \%)$ & $3(6.2 \%)$ \\
& $25-50 \%$ (Score 2) & $3(30 \%)$ & $2(14.2 \%)$ & $5(35.7 \%)$ & $2(12.5 \%)$ \\
& $>50 \%$ (Score 3) & $5(50 \%)$ & $7(50 \%)$ & $8(57.1 \%)$ & $11(6.9 \%)$ \\
\hline Average & & 51.8 & 62.3 & 69.2 & 41.7 \\
\hline - value* & 0.043 & & & & \\
\hline
\end{tabular}

*Tested with Anova.

phospholipase C gamma (PLC $\gamma$ ) - protein kinase C (PKC) - Raf kinase - MEK - mitogen-activated protein kinase (MAPK) pathway and the phosphatidylinositol 3' - kinase (PI3K) pathway that triggers changes in actin cytoskeleton and induces cell migration.(8)

VEGF expressions in this study varied at each $\mathrm{T}$ stage. There are several factors that can regulate VEGF expression, including hypoxia, free radicals, $\mathrm{pH}$ imbalance, and nutrient deficiencies.(15) To compensate the hypoxia and nutrient deficiencies and to escape from tumor necrosis, tumor cells express hypoxia-inducible factors (HIF), which will activate the transcription of over 40 genes, including VEGF. (9) Suitable with the previous study, there was a positive correlation between HIF- $1 \alpha$ and VEGF immunoreactivities $(p<0.001)$ in urothelial carcinoma.(16) VEGF and VEGF receptor transcription are directly activated by HIF-1 $\alpha$ by binding hypoxia response element (HRE) and plays an important role during normal growth and tumor formation. (17) In addition of hypoxia, conditions of $\mathrm{pH}$ imbalance and high temperature can also affect the level of VEGF transcription and VEGF expression through observation of nitric oxide (NO) donor sodium nitroprusside (SNP) and hydrogen peroxide.(15) These factors may have more direct role in VEGF expression than their relation to T stage, and these factors have not been analyzed in this study.

The different expression of MMP-9 on each T stage indicate a difference in expression of MMP-9 on the depth of tumor cell invasion. This is in accordance with the previous
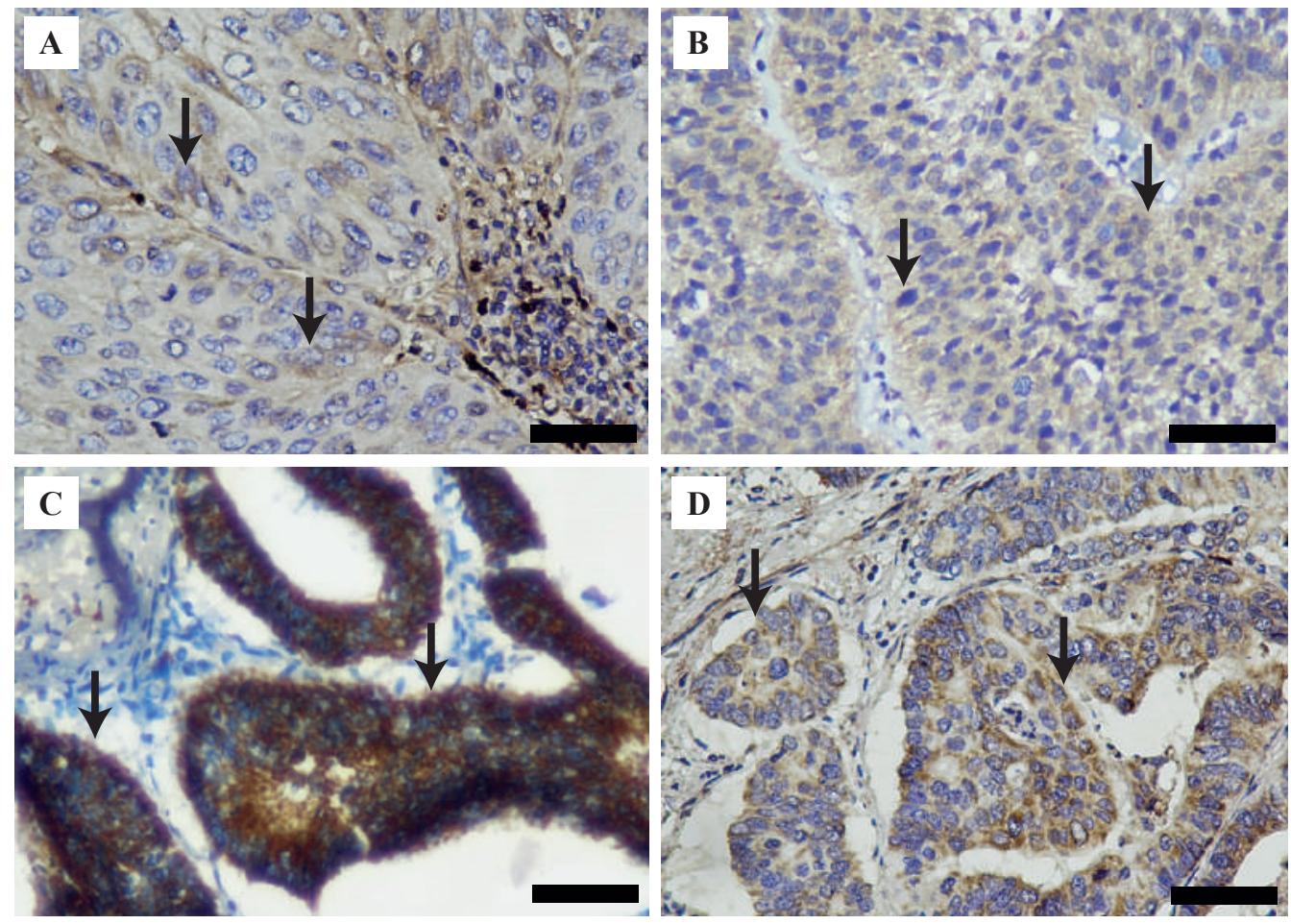

Figure 2. Immunohistochemical expression of MMP-9 expression in various $\mathbf{T}$ stage of bladder urothelial carcinoma. A: T1 stage, $70 \%$ tumor cells were stained; B: T2 stage, 90\% tumor cells were stained; C: T3 stage, 100\% tumor cells were stained; D: T4 stage, 35\% tumor cells were stained. Black bar: $5 \mu \mathrm{m}$. 
Table 5. Comparison of MMP-9 expression between two group.

\begin{tabular}{cc}
\hline T Stage & $\boldsymbol{p}$-value \\
\hline T1-T2 & 0.353 \\
T1-T3 & 0.127 \\
T1-T4 & 0.364 \\
T2-T3 & 0.504 \\
T2-T4 & 0.044 \\
T3-T4 & 0.008 \\
\hline
\end{tabular}

research which conducted research on MMP-9 expression in the progression of urothelial bladder carcinoma and obtained an increase in MMP-9 expression is in line with increased tumor invasion. $(18,19)$ Likewise, in the prior study which compared normal bladder tissue and bladder urothelial carcinoma, they found an increase in MMP9 expression (up to 50\%) in bladder urothelial carcinoma compared with normal bladder tissue.(20)

MMP-9 is involved in several biological processes as a proteolytic that degrades extracellular matrix, separator between cells, separator of interactions between cells and extracellular matrix, division of proteins on cell surfaces, division of proteins in the extracellular environment.(21) MMP-9 which is secreted by tumor cells is related to the ability to metastasize, this is because MMP-9 can degrade collagen types IV, V, VII and X. Type IV collagen is the main collagen supporting the basal membrane and is especially abundant in vascular endothelium in basal membrane, and acts as a barrier to invasion and metastasis. The high expression of MMP-9 causes degradation of extracellular matrix making it easier for tumor cells to invade. $(22,23)$

The correlation between VEGF expression and MMP9 expression in T-stage urothelial bladder carcinoma showed a significant result where the higher VEGF expression, the higher the MMP-9 expression in invasion bladder urothelial carcinoma (Figure 3). The results of this study are similar with previous study which stated a correlation between VEGF and MMP-9 in retinoblastoma tumor invasion.(24) The basis of angiogenesis is the migration of endothelial cells into the surrounding loose tissue. MMP-9 has complex roles including remodelling of extracellular matrix, and is activated by angiogenic factors, one of which is VEGF. Conversely, MMP-9 also promotes endothelial cell migration and triggers angiogenic switches (a balance effect between proangiogenic and anti-angiogenic) by releasing VEGF during the carcinogenesis process.(11,25)

The limitation of this study was the small number of samples which originating from only one hospital thus making us use total sampling procedure.

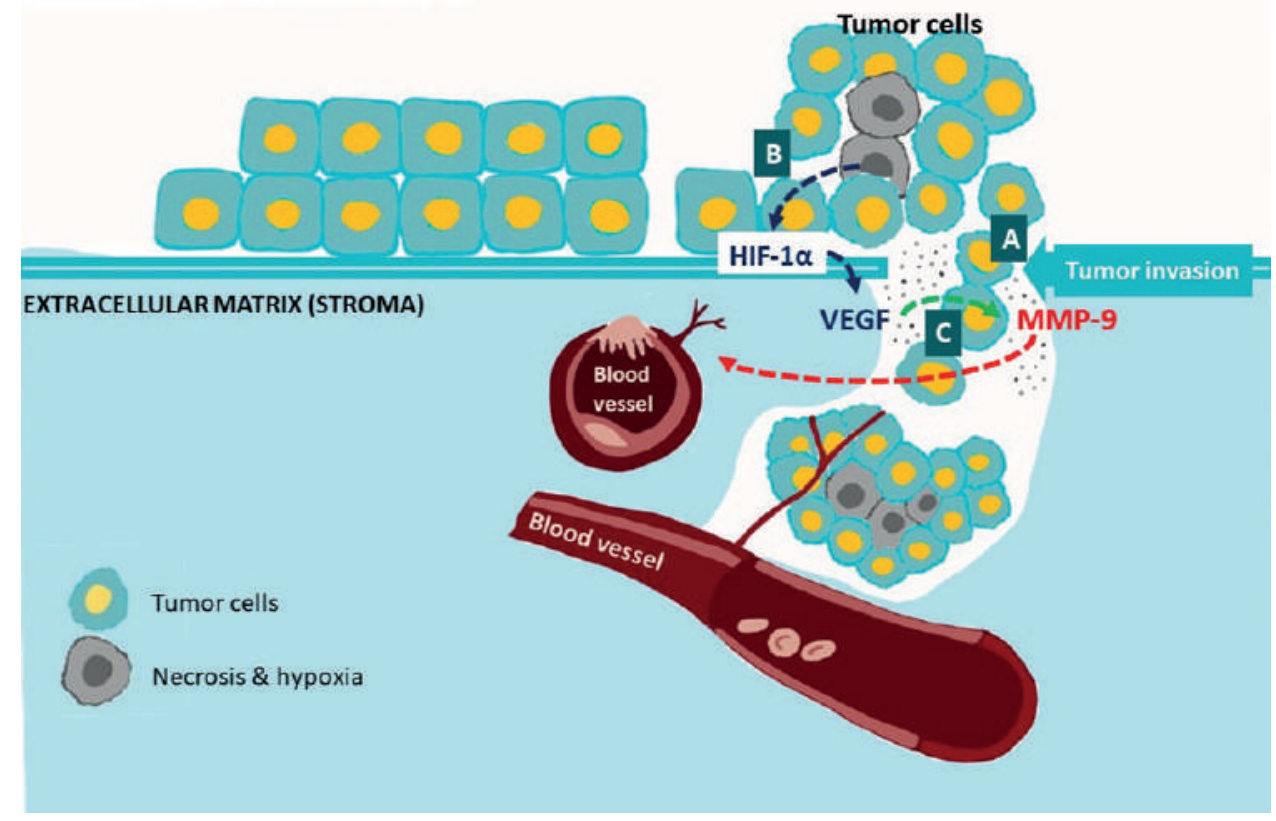

Figure 3. VEGF and MMP-9 expression. A: The invasion process begins with the destruction of the basement membrane and extracellular matrix by the proteolytic enzyme MMP9 secreted by tumor cells; B: The uncontrolled proliferation of tumor cells results a lack of nutrient and oxygen supply to tumor cells. To prevent necrosis, tumor cells express HIF $1 \alpha$. HIF $1 \alpha$ then triggers the release of VEGF which is the main mediator of tumor angiogenesis; C: At the same time, MMP9 secretion is also triggered by VEGF to support the angiogenesis process by degrading the extracellular matrix around blood vessels. 


\section{Conclusion}

MMP-9 expression is different on each depth of invasion in bladder urothelial carcinoma. The significant correlation of VEGF and MMP-9 expression in bladder urothelial carcinoma may prove the synergistically role of both proteins in tumor invasion by MMP-9 degradation extracellular matrix.

\section{Acknowledgements}

Thanks to Dr. Budi Utomo, dr., M.Kes. who helped us in the statistical analysis also to all the lecturers in Department of Anatomical Pathology, Faculty of Medicine, Universitas Airlangga for the guidance in finishing this study.

\section{Authors Contribution}

All authors were involved in concepting and planning the research, performed the data acquisition/collection, calculated the experimental data and performed the analysis, drafted the manuscript and designed the figures, interpreted the results and took parts in giving critical revision of the manuscript.

\section{References}

1. Bray F, Ferlay J, Soerjomataram I, Siegel R, Torre L, Jemal A. Global cancer statistics 2018: GLOBOCAN estimates of incidence and mortality worldwide for 36 cancers in 185 countries. Ca Cancer J Clin. 2018; 68: 394-424.

2. Giridhar KV, Kohli M. Management of muscle-invasive urothelial cancer and the emerging role of immunotherapy in advanced urothelial cancer. Mayo Clin Proc. 2017; 92: 1564-82.

3. Tanaka MF, Sonpavde G. Diagnosis and management of urothelial carcinoma of the bladder diagnosis and management of urothelial carcinoma of the bladder. J Postgrad Med. 2015; 123: 43-55.

4. Zhao M, He XL, Teng XD. Understanding the molecular pathogenesis and prognostics of bladder cancer : an overview. Chin J Cancer Res. 2016; 28: 92-8.

5. Inamura K. Bladder cancer: new insights into its molecular pathology. Cancers. 2018; 10: 100. doi: 10.3390/cancers 10040100.

6. Magers MJ, Lopez-Beltran A, Montironi R, Williamson SR, Kaimakliotis HZ, Cheng L. Staging of bladder cancer. Histopathology. 2019; 74: 112-34.

7. Magalhaes A, Dias S. Angiogenesis-vessels recruitment by tumor cells. In: Fior R, Zilhão R, editors. Molecular and Cell Biology of Cancer. Freiburg: Springer Nature; 2019. p.141-57.

8. Kopparapu PK, Boorjian SA, Robinson BD, Downes M, Gudas LJ, Mongan NP, et al. Expression of VEGF and its receptors VEGFR1/ VEGFR2 is associated with invasiveness of bladder cancer. Anticancer Res. 2013; 33: 2381-90.

9. Bronsert P, Werner M. Pathology of Tumor Angiogenesis. Freiburg: Springer Nature; 2018

10. Fus LP, Gornicka B. Role of angiogenesis in urothelial bladder carcinoma. Cent European J Urol. 2016; 69: 258-63.

11. Amalinei C, Caruntu ID, Giuşca SE, Balan RA. Matrix metalloproteinases involvement in pathologic conditions. Rom J Morphol Embryol. 2010; 51: 215-28.

12. Gunes M, Kemik AS, Pirincci N, Gecit I, Taken K, Yuksel MB, et al. Preoperative levels of matrix metalloproteinase-7 and-9 and tissue inhibitor of matrix metalloproteinase-1 relation to pathologic parameters in bladder carcinoma patients. Asian Pac J Cancer Prev. 2013; 14: 873-6.

13. Al-Bassam SSA, Kadhim HS, Khashman BM. Possible association of vascular endothelial growth factor with grades of breast cancer. Int J Res Pharm Chem. 2014; 4: 291-3.

14. Ali $\mathrm{HH}$, Ibraheem $\mathrm{MH}$, Alhindawi MM. Immunohistochemical expression of matrix metalloproteinase- 9 in urothelial carcinoma of urinary bladder. Kasr Al Ainy Med J. 2018; 24: 109-14.

15. Wang F, Xu P, Xie KC, Chen XF, Li CY, Huang Q. Effects of tumor microenviromental factors on VEGF expression. Biomed Rep. 2013; 1: 539-44.

16. Deniz H, Karakök M, Yagcı F, Güldür ME. Evaluation of relationship between HIF-1 $\alpha$ immunoreactivity and stage, grade, angiogenic profile and proliferative index in bladder urothelial carcinomas. Int Urol Nephrol. 2010; 42: 103-7.

17. Yang Y, Sun M, Wang L, Jiao B. HIFs, angiogenesis, and cancer. J Cell Biochem. 2013; 114: 967-74.

18. Reis ST, Leite KRM, Piovesan LF, Pontes-Junior J, Viana NI, Abe DK, et al. Increased expression of MMP-9 and IL-8 are correlated with poor prognosis of bladder cancer. BMC Urol. 2012; 12: 18. doi: 10.1186/1471-2490-12-18.

19. Tang Z, Tang C, Juanfen MO, Ying X. Role of MMPs expression in the invasion and metastasis of bladder cancer. Chin J Primary Med Pharm. 2018; 25: 156-60.

20. Wu GJ, Bao JS, Yue ZJ, Zeng FC, Cen S, Tang ZY, et al. Elevated expression of matrix metalloproteinase- 9 is associated with bladder cancer pathogenesis. J Cancer Res Ther. 2018; 14: 54-9.

21. Huang H. Matrix metalloproteinase-9 (MMP-9) as a cancer biomarker and MMP-9 biosensors: recent advances. Sensors. 2018; 18: 3249. doi: 10.3390/s18103249.

22. Vandooren J, Van den Steen PE, Opdenakker G. Biochemistry and molecular biology of gelatinase B or matrix metalloproteinase-9 (MMP-9): the next decade. Crit Rev Biochem Mol Biol. 2013; 48: 222-72.

23. Conlon GA, Murray GI. Recent advances in understanding the roles of matrix metalloproteinases in tumour invasion and metastasis. J Pathol. 2019; 247: 629-40

24. Wijayanti E, Fauzyah D. Correlation between VEGF and MMP-9 expression in retinoblastoma extraocular invasion. Majalah Patologi Indonesia. 2017; 26: 44-9.

25. Quintero-Fabián S, Arreola R, Becerril-Villanueva E, TorresRomero JC, Arana-Argáez VE, Lara-Riegos J, et al. Role of matrix metalloproteinases in angiogenesis and cancer. Front Oncol. 2019; 9: 1370 . doi: $10.3389 /$ fonc. 2019.01370 . 